\title{
Jordan triple mappings on positive definite matrices
}

\author{
Eszter Gselmann
}

\begin{abstract}
The main aim of this paper is to characterize the determinant function on the set of positive definite $n \times n$ matrices with entries from $\mathbb{F}$.
\end{abstract}

Mathematics Subject Classification (2010). Primary 39B42; Secondary 15A30.

Keywords. Jordan triple mapping, determinant, characterization, positive definite matrix.

\section{Introduction and preliminaries}

The characterization of the determinant function is one of the oldest question in the field of linear algebra. The standard approach to the study of determinants is to prove the existence, for any $n$-dimensional vector space $X$ over a field $\mathbb{F}$, of a nonzero alternating multilinear form $d: X^{n} \rightarrow \mathbb{F}$ and to define the determinant function det: $\mathscr{L}(X) \rightarrow \mathbb{F}$ by the requirement

$$
\operatorname{det}(T) \cdot d\left(x_{1}, \ldots, x_{n}\right)=d\left(T x_{1}, \ldots, T x_{n}\right) \quad(T \in \mathscr{L}(X))
$$

for all $\left(x_{1}, \ldots, x_{n}\right) \in X^{n}$. These conditions determine $d$ up to a multiplicative constant and hence determine the function det. See e.g. Fearnley-Sander $[5]$.

It would be however desirable to characterize the determinant function through a certain identity. For instance, it is well-known that the determinant function fulfills the identity

$$
\chi(A \cdot B)=\chi(A) \cdot \chi(B)
$$

and also the equation

$$
\psi(A \cdot B \cdot A)=\psi(A) \cdot \psi(B) \cdot \psi(A)
$$

This research has been supported by the Hungarian Scientific Research Fund (OTKA) Grant NK 81402 and by the 'Lendület' Program (LP2012-46/2012) of the Hungarian Academy of Sciences. 
for all $A, B \in \mathscr{L}(X)$. Mappings $\psi: \mathscr{L}(X) \rightarrow \mathbb{F}$ satisfying the previous equation are termed to be Jordan triple mappings.

The main aim of this paper is to characterize the determinant function on the set of positive definite $n \times n$ matrices with entries from $\mathbb{F}$. In this setting, equation (1) is obviously inappropriate, since the ordinary product of two positive definite matrices will not be in general a positive definite matrix. However, the Jordan triple product of two such matrix will be again positive. Thus, in what follows we will investigate equation (2), or more precisely, its additive counterpart, namely the following equation

$$
\phi(A \cdot B \cdot A)=2 \phi(A)+\phi(B) .
$$

The notion of Jordan triple mappings were firstly introduced in Yamaguti [12. Further, the characterization of such type of mappings began in the 1960's, see for example Gáspár [6], Hitotumatu [7, Kurepa [9]. Concerning Jordan triple mappings, the results are rather recent, see Dobovišek 4, Bobecka-Wesołowski [3] and Molnár [11.

In what follows, we will shortly review the known results in this area. In [3] differentiable Jordan triple mappings $\left.\phi: \mathscr{P}_{n}(\mathbb{R}) \rightarrow\right] 0,+\infty[$ were determined.

Theorem 1 (Bobecka-Wesołowski). Let $\left.\phi: \mathscr{P}_{n}(\mathbb{R}) \rightarrow\right] 0,+\infty[$ be a differentiable mapping so that

$$
\phi(\sqrt{Y} X \sqrt{Y})=\phi(X) \phi(Y)
$$

is fulfilled for all $X, Y \in \mathscr{P}_{n}(\mathbb{R})$. Then there exists $\kappa \in \mathbb{R}$ so that

$$
\phi(X)=\operatorname{det}(X)^{\kappa} \quad\left(X \in \mathscr{P}_{n}(\mathbb{R})\right) .
$$

Later, using a theorem of Gleason, it was showed that differentiability can be weakened and continuous Jordan triple mappings $\phi: \mathscr{P}_{n}(\mathbb{C}) \rightarrow \mathbb{R}$ were completely described in $[1]$, assuming that $n \geq 3$. This method however does not work in case $\mathscr{P}_{2}(\mathbb{C})$.

Theorem 2 (Molnár). Let $n \geq 3$ be arbitrarily fixed and $\phi: \mathscr{P}_{n}(\mathbb{C}) \rightarrow \mathbb{R}$ be a continuous functional. Assume that for all $X, Y \in \mathscr{P}_{n}(\mathbb{C})$ functional equation

$$
\phi(Y X Y)=\phi(Y) \phi(X) \phi(Y)
$$

is fulfilled. Then either $\phi$ is identically zero or there exists $\kappa \in \mathbb{R}$ such that

$$
\phi(X)=\operatorname{det}(X)^{\kappa} \quad\left(X \in \mathscr{P}_{n}(\mathbb{R})\right) .
$$

Without any regularity assumption, Jordan triple mappings were found out in [4], though not for mappings $\phi: \mathscr{P}_{n}(\mathbb{F}) \rightarrow \mathbb{R}$ but for $\phi: \mathscr{M}_{n}(\mathbb{F}) \rightarrow \mathbb{R}$. Our main aim is to investigate Jordan triple mappings in their natural setting. More precisely, we would like to solve functional equation

$$
\phi(Y X Y)=2 \phi(Y)+\phi(X) \quad\left(X, Y \in \mathscr{P}_{n}(\mathbb{F})\right)
$$

for the unknown function $\phi: \mathscr{P}_{n}(\mathbb{F}) \rightarrow \mathbb{R}$ without any regularity supposition and irrespective of the value $n$. 
Firstly we will fix the notation and the terminology that will be used subsequently. Concerning the theory of functional equations, we refer to the two basic monographs Aczél [1] and Kuczma [8].

Definition 3. Let $A \subset] 0,+\infty[$ be a nonempty set and

$$
\mathscr{L}=\{(x, y) \in] 0,+\infty\left[^{2} \mid x, y, x \cdot y \in A\right\} .
$$

A function $\ell: A \rightarrow \mathbb{R}$ is termed to be a logarithmic function on $A$ if

$$
\ell(x y)=\ell(x)+\ell(y)
$$

is fulfilled for any $(x, y) \in \mathscr{L}$. In case $A=] 0,+\infty[$, then the function $\ell$ will simply be called logarithmic.

In what follows $\mathbb{R}, \mathbb{C}$ will stand for the set of the real, respectively complex numbers.

Definition 4. Let $\mathbb{F} \in\{\mathbb{R}, \mathbb{C}\}$ and $n \in \mathbb{N}$ be fixed, then

$$
\mathscr{M}_{n}(\mathbb{F})=\{X \mid X \text { is an } n \times n \text { matrix with entries from } \mathbb{F}\}
$$

and

$$
\mathscr{P}_{n}(\mathbb{F})=\left\{X \in \mathscr{M}_{n}(\mathbb{F}) \mid X \text { is positive definite }\right\} .
$$

Henceforward, concerning matrix analysis, the notions and results of the basic monograph Bhatia [2] will be utilized. The following easy statements will be used permanently.

Lemma 1. Let $n \in \mathbb{N}$ and $F \in\{\mathbb{R}, \mathbb{C}\}$. Then

(i) $X \in \mathscr{P}_{n}(\mathbb{F})$ if and only if $X$ is Hermitian and all its principal minors are positive.

(ii) $X \in \mathscr{P}_{n}(\mathbb{F})$ if and only if all its eigenvalues are positive.

(iii) $X \in \mathscr{P}_{n}(\mathbb{F})$ if and only if there exists $Y \in \mathscr{P}_{n}(\mathbb{F})$ such that

$$
X=Y^{2} \text {, }
$$

such $Y$ is uniquely determined. We will write $Y=\sqrt{X}$ and call it the square root of $X$.

\section{The $2 \times 2$ case}

At first we prove the following. Henceforth we always assume $\mathbb{F} \in\{\mathbb{R}, \mathbb{C}\}$.

Theorem 5. Let $\phi: \mathscr{P}_{2}(\mathbb{F}) \rightarrow \mathbb{R}$ be a mapping for which

$$
\phi(Y X Y)=\phi(X)+2 \phi(Y)
$$

holds for all $X, Y \in \mathscr{P}_{2}(\mathbb{F})$. Then, there exists a logarithmic function $l$ : ] $0,+\infty[\rightarrow \mathbb{R}$ such that

$$
\phi(X)=l(\operatorname{det}(X)) \quad\left(X \in \mathscr{P}_{2}(\mathbb{F})\right) .
$$


Proof. Let $X, Y \in \mathscr{P}_{2}(\mathbb{F})$ be diagonal matrices, then obviously $X \cdot Y=Y \cdot X$, therefore

$$
\phi\left(X \cdot Y^{2}\right)=\phi(X)+2 \phi(Y) .
$$

If we replace $Y$ by $\sqrt{Y}$, we obtain that

$$
\phi(X \cdot Y)=\phi(X)+\phi(Y)
$$

is valid for all diagonal matrices $X, Y \in \mathscr{P}_{2}(\mathbb{F})$. Let $X, Y \in \mathscr{P}_{2}(\mathbb{F})$ be diagonal matrices, say,

$$
X=\left(\begin{array}{cc}
\lambda_{1} & 0 \\
0 & \lambda_{2}
\end{array}\right) \quad \text { and } \quad Y=\left(\begin{array}{cc}
\mu_{1} & 0 \\
0 & \mu_{2}
\end{array}\right),
$$

where $\lambda_{1}, \lambda_{2}, \mu_{1}, \mu_{2}$ are positive real numbers. Then

$$
X \cdot Y=\left(\begin{array}{cc}
\lambda_{1} \mu_{1} & 0 \\
0 & \lambda_{2} \mu_{2}
\end{array}\right)
$$

Define the function $\varphi:] 0,+\infty\left[{ }^{2} \rightarrow \mathbb{R}\right.$ by

$$
\varphi\left(\lambda_{1}, \lambda_{2}\right)=\phi\left(\begin{array}{cc}
\lambda_{1} & 0 \\
0 & \lambda_{2}
\end{array}\right) \quad\left(\lambda_{1}, \lambda_{2} \in\right] 0,+\infty[) .
$$

Then due to identity $(*)$, we have

$$
\varphi\left(\lambda_{1} \mu_{1}, \lambda_{2} \mu_{2}\right)=\varphi\left(\lambda_{1}, \lambda_{2}\right)+\varphi\left(\mu_{1}, \mu_{2}\right) \quad\left(\lambda_{1}, \lambda_{2}, \mu_{1}, \mu_{2} \in\right] 0,+\infty[) .
$$

With the choice $\lambda_{2}=\mu_{1}=1$ and $\mu_{2}=\lambda_{2}$ this equation yields that

$$
\varphi\left(\lambda_{1}, \lambda_{2}\right)=\varphi\left(\lambda_{1}, 1\right)+\varphi\left(1, \lambda_{2}\right)
$$

holds for all $\left.\lambda_{1}, \lambda_{2} \in\right] 0,+\infty$ [. On the other hand (*) with $\lambda_{2}=\mu_{2}=1$ implies that

$$
\varphi\left(\lambda_{1} \mu_{1}, 1\right)=\varphi\left(\lambda_{1}, 1\right)+\varphi\left(\mu_{1}, 1\right) \quad\left(\lambda_{1}, \mu_{1} \in\right] 0,+\infty[),
$$

further, identity $(*)$ with the choice $\lambda_{1}=\mu_{1}=1$ yields that

$$
\varphi\left(1, \lambda_{2} \mu_{2}\right)=\varphi\left(1, \lambda_{2}\right)+\varphi\left(1, \mu_{2}\right) \quad\left(\lambda_{2}, \mu_{2} \in\right] 0,+\infty[) .
$$

All in all, this means that there exist logarithmic functions $\left.l_{1}, l_{2}:\right] 0,+\infty[\rightarrow \mathbb{R}$ such that

$$
l_{1}(\lambda)=\varphi(\lambda, 1) \quad \text { and } \quad l_{2}(\lambda)=\varphi(1, \lambda) \quad(\lambda \in] 0,+\infty[)
$$

is fulfilled. Therefore for all $\lambda, \mu \in] 0,+\infty[$

$$
\phi\left(\begin{array}{cc}
\lambda & 0 \\
0 & \mu
\end{array}\right)=\varphi(\lambda, \mu)=l_{1}(\lambda)+l_{2}(\mu)
$$

is satisfied.

Let now $X \in \mathscr{P}_{2}(\mathbb{F})$

$$
X=\left(\begin{array}{ll}
a & b \\
\bar{b} & d
\end{array}\right)
$$

be arbitrary. Then

$$
Y \cdot X \cdot Y=\left(\begin{array}{cc}
1 & \frac{b}{\sqrt{a} \sqrt{d}} \\
\frac{\bar{b}}{\sqrt{a} \sqrt{d}} & 1
\end{array}\right),
$$


where

$$
Y=\left(\begin{array}{cc}
\frac{1}{\sqrt{a}} & 0 \\
0 & \frac{1}{\sqrt{d}}
\end{array}\right) .
$$

Let $U=\{x \in \mathbb{F}:\|x\|<1\}$ and define the function $f: U \rightarrow \mathbb{R}$ through

$$
f(x)=\phi\left(\begin{array}{ll}
1 & x \\
\bar{x} & 1
\end{array}\right) .
$$

Let

$$
X=\left(\begin{array}{cc}
1 & x \\
\bar{x} & 1
\end{array}\right) \quad(x \in U)
$$

be arbitrarily fixed. Then

$$
\phi\left(X^{-1}\right)=-\phi(X)=-f(x) .
$$

On the other hand,

$$
\begin{aligned}
& \phi\left(X^{-1}\right)=\phi\left(\begin{array}{cc}
-\frac{1}{x^{2}-1} & \frac{x}{x^{2}-1} \\
\frac{x}{x^{2}-1} & -\frac{1}{x^{2}-1}
\end{array}\right) \\
&=\phi\left[\left(\begin{array}{cc}
\frac{1}{\sqrt{1-x^{2}}} & 0 \\
0 & \frac{1}{\sqrt{1-x^{2}}}
\end{array}\right) \cdot\left(\begin{array}{cc}
1 & -x \\
-x & 1
\end{array}\right) \cdot\left(\begin{array}{cc}
\frac{1}{\sqrt{1-x^{2}}} & 0 \\
0 & \frac{1}{\sqrt{1-x^{2}}}
\end{array}\right)\right] \\
&=-l\left(\sqrt{1-x^{2}}\right)+f(-x)-l\left(\sqrt{1-x^{2}}\right) .
\end{aligned}
$$

All in all, this implies that

$$
f(x)+f(-x)=2 l\left(\sqrt{1-x^{2}}\right) \quad(x \in U) .
$$

From this it follows however that

$$
f(x)=\frac{1}{2} l\left(1-x^{2}\right)+g(x) \quad(x \in U),
$$

where $g: U \rightarrow \mathbb{R}$ is an odd function.

Due to the definition of the mapping $f$, we also have

$$
\begin{array}{r}
f(x)+2 f(y)=\phi\left(\begin{array}{ll}
1 & x \\
\bar{x} & 1
\end{array}\right)+2 \phi\left(\begin{array}{ll}
1 & y \\
\bar{y} & 1
\end{array}\right)=\phi\left(\left(\begin{array}{ll}
1 & y \\
\bar{y} & 1
\end{array}\right) \cdot\left(\begin{array}{ll}
1 & x \\
\bar{x} & 1
\end{array}\right) \cdot\left(\begin{array}{ll}
1 & y \\
\bar{y} & 1
\end{array}\right)\right) \\
=\phi\left(\begin{array}{rl}
\frac{y^{2}+2 x y+1}{x y^{2}+2 y+x} & x y^{2}+2 y+x \\
y^{2}+2 x y+1
\end{array}\right)
\end{array}
$$


holds for all $x, y \in U$. On the other hand, for all $x, y \in U$,

$$
\begin{aligned}
\phi\left(\begin{array}{cc}
\frac{y^{2}+2 x y+1}{x y^{2}+2 y+x} & x y^{2}+2 y+x \\
y^{2}+2 x y+1
\end{array}\right) \\
=\phi\left(\left(\begin{array}{cc}
\sqrt{y^{2}+2 x y+1} & 0 \\
0 & \sqrt{y^{2}+2 x y+1}
\end{array}\right) .\right. \\
\\
\left.\quad\left(\begin{array}{ccc}
\frac{1}{\frac{x y^{2}+2 y+x}{y^{2}+2 x y+1}} & \frac{x y^{2}+2 y+x}{y^{2}+2 x y+1} \\
1
\end{array}\right) \cdot\left(\begin{array}{cc}
\sqrt{y^{2}+2 x y+1} & 0 \\
0 & \sqrt{y^{2}+2 x y+1}
\end{array}\right)\right) \\
2\left\{l_{1}\left(\sqrt{y^{2}+2 x y+1}\right)+l_{2}\left(\sqrt{y^{2}+2 x y+1}\right)\right\}+f\left(\frac{x y^{2}+2 y+x}{y^{2}+2 x y+1}\right) .
\end{aligned}
$$

Thus

$$
\begin{aligned}
f(x)+2 f(y)=2\left\{l_{1}\left(\sqrt{y^{2}+2 x y+1}\right)\right. & \left.+l_{2}\left(\sqrt{y^{2}+2 x y+1}\right)\right\} \\
+ & f\left(\frac{x y^{2}+2 y+x}{y^{2}+2 x y+1}\right) \quad(x, y \in U) .
\end{aligned}
$$

Since the functions $l_{1}$ and $l_{2}$ are logarithmic, this yields that

$$
f(x)+2 f(y)=l\left(y^{2}+2 x y+1\right)+f\left(\frac{x y^{2}+2 y+x}{y^{2}+2 x y+1}\right)
$$

is fulfilled for any $x, y \in U$, where $l=l_{1}+l_{2}$. If we replace here $x$ and $y$ by $-x$ and $-y$, respectively and subtract the equations side by side,

$$
g\left(\frac{x^{2} y+2 x+y}{2 x y+x^{2}+1}\right)=2 g(x)+g(y)
$$

can be derived.

Thus, if $A \in \mathscr{P}_{2}(\mathbb{F})$ and

$$
A=\left(\begin{array}{ll}
a & b \\
\bar{b} & d
\end{array}\right)
$$

then

$$
A=\left(\begin{array}{cc}
\sqrt{a} & 0 \\
0 & \sqrt{d}
\end{array}\right) \cdot\left(\begin{array}{cc}
1 & \frac{b}{\sqrt{a d}} \\
\frac{\bar{b}}{\sqrt{a d}} & 1
\end{array}\right) \cdot\left(\begin{array}{cc}
\sqrt{a} & 0 \\
0 & \sqrt{d}
\end{array}\right)
$$

therefore,

$$
\begin{aligned}
\phi(A)=2 \phi\left(\begin{array}{cc}
\sqrt{a} & 0 \\
0 & \sqrt{d}
\end{array}\right)+\phi\left(\begin{array}{cc}
1 & \frac{b}{\sqrt{a d}} \\
\frac{b}{\sqrt{a d}} & 1
\end{array}\right) & \\
& =l_{1}(a)+l_{2}(d)+\frac{1}{2} l\left(1-\frac{b^{2}}{a d}\right) .
\end{aligned}
$$

Equivalently,

$$
\phi(A)=l(\operatorname{det}(A))+g\left(\frac{b}{\sqrt{a d}}\right)+k\left(\frac{d}{a}\right)
$$


is satisfied for any $A=\left(\begin{array}{ll}a & b \\ b & d\end{array}\right) \in \mathscr{P}_{2}(\mathbb{F})$. Obviously, the mapping

$$
\mathscr{P}_{2}(\mathbb{F}) \ni A \longmapsto l(\operatorname{det}(A))
$$

is a Jordan triple mapping. This means that the function

$$
\chi(A)=\chi\left(\begin{array}{ll}
a & b \\
b & d
\end{array}\right)=g\left(\frac{b}{\sqrt{a d}}\right)+k\left(\frac{d}{a}\right) \quad\left(A=\left(\begin{array}{ll}
a & b \\
b & d
\end{array}\right) \in \mathscr{P}_{2}(\mathbb{F})\right)
$$

also has to be a Jordan triple mapping. Due to the properties of the functions $g$ and $k$,

$$
k\left(\frac{d}{a}\right)=\frac{\chi\left(\begin{array}{ll}
a & b \\
b & d
\end{array}\right)+\chi\left(\begin{array}{cc}
a & -b \\
-b & d
\end{array}\right)}{2}
$$

yielding that $k$ is identically zero, in other words

$$
l_{1}(x)=l_{2}(x) \quad(x \in] 0,+\infty[) .
$$

All in all, we known that the mapping $\chi: \mathscr{P}_{n}(\mathbb{F}) \rightarrow \mathbb{R}$ is a Jordan triple mapping and

$$
\chi\left(\begin{array}{ll}
a & b \\
b & d
\end{array}\right)=g\left(\frac{b}{\sqrt{a d}}\right)
$$

is valid. From this $\chi \equiv 0$ can de concluded.

Definition 6. A function $f: \mathscr{P}_{2}(\mathbb{F}) \rightarrow \mathbb{R}$ is called regular if at least one of the following statements are valid.

(i) The function $f$ is continuous at a point $X_{0} \in \mathscr{P}_{2}(\mathbb{F})$.

(ii) There exists a set $A \subset \mathscr{P}_{2}(\mathbb{F})$ of positive Lebesgue measure such that the function $f$ is bounded above or below on $A$;

(iii) There exists a set $A \subset \mathscr{P}_{2}(\mathbb{F})$ of positive Lebesgue measure such that the restriction of $f$ to the set $A$ is measurable (in the sense of Lebesgue).

The following lemma shows that every logarithmic function $\ell:] 0,+\infty[\rightarrow$ $\mathbb{R}$ can be expressed with the aid of additive functions, see [8, Theorem 13.1.2.].

Lemma 2. Let $\ell:] 0,+\infty[\rightarrow \mathbb{R}$ be a logarithmic function. Then there exists an additive function $a: \mathbb{R} \rightarrow \mathbb{R}$ such that

$$
\ell(x)=a(\ln (x)) \quad(x \in \mathbb{R}) .
$$

With the aid of the previous lemma the following statement can be derived for regular logarithmic functions.

Theorem 7. Let $\ell:] 0,+\infty[\rightarrow \mathbb{R}$ be a regular logarithmic function. Then there exists $\kappa \in \mathbb{R}$ such that

$$
\ell(x)=\kappa \ln (x) \quad(x \in] 0,+\infty[) .
$$

Theorem 8. Let $\phi: \mathscr{P}_{2}(\mathbb{F}) \rightarrow \mathbb{R}$ be a mapping for which

$$
\phi(Y X Y)=\phi(X)+2 \phi(Y)
$$


holds for all $X, Y \in \mathscr{P}_{2}(\mathbb{F})$. If the function $\phi$ is regular, then there exists $\kappa \in \mathbb{R}$ such that

$$
\phi(X)=\kappa \ln (\operatorname{det}(X))
$$

holds for all $X \in \mathscr{P}_{2}(\mathbb{F})$.

Proof. From the previous theorem we immediately get that there exists a logarithmic function $l:] 0,+\infty[\rightarrow \mathbb{R}$ such that

$$
\phi(X)=l(\operatorname{det}(X)) \quad\left(X \in \mathscr{P}_{2}(\mathbb{F})\right) .
$$

Additionally, we know that the function $\phi$ is regular. Thus the mapping

$$
\mathscr{P}_{2}(\mathbb{F}) \ni X \longmapsto l(\operatorname{det}(X))
$$

is regular. From this however the regularity of $l$ follows. Thus, there exists a constant $\kappa \in \mathbb{R}$ so that

$$
\phi(X)=\kappa \ln (\operatorname{det}(X))
$$

is satisfied for all $X \in \mathscr{P}_{2}(\mathbb{F})$.

\section{The general case}

In view of the results of the previous section, we are able to prove our main theorem.

Theorem 9. Let $n \in \mathbb{N}$ and assume that for the mapping $\phi: \mathscr{P}_{n}(\mathbb{F}) \rightarrow \mathbb{R}$

$$
\phi(Y X Y)=\phi(X)+2 \phi(Y)
$$

holds for all $X, Y \in \mathscr{P}_{n}(\mathbb{F})$. Then there is a logarithmic function $\left.l:\right] 0,+\infty[$ $\rightarrow \mathbb{R}$ so that

$$
\phi(X)=l(\operatorname{det}(X))
$$

is fulfilled for any $X \in \mathscr{P}_{n}(\mathbb{F})$.

Proof. In view of the results of the previous section, the statement of the theorem is valid for $n=2$.

The remaining part of the proof will be divided into two parts.

Part I. We will show that assuming that the statement is true for a certain $n \in \mathbb{N}$, we will show that the statement is also valid for $2 n$.

Let us assume that the statement of the theorem is fulfilled for a certain $n \in \mathbb{N}$.

Clearly, if the matrices $X, Y \in \mathscr{P}_{2 n}(\mathbb{F})$ commute, then

$$
\phi(Y X Y)=\phi\left(X Y^{2}\right),
$$

so, the equation for $\phi$ simply yields that

$$
\phi(X Y)=\phi(X)+\Phi(Y),
$$

since $\Phi\left(Y^{2}\right)=2 \phi(Y)$.

Let now

$$
X=\left(\begin{array}{l|l}
A & \mathbf{0} \\
\hline \mathbf{0} & I
\end{array}\right) \quad \text { and } \quad Y=\left(\begin{array}{c|c}
I & \mathbf{0} \\
\hline \mathbf{0} & D
\end{array}\right),
$$


where $A, D \in \mathscr{P}_{n}(\mathbb{F})$ and $I \in \mathscr{P}_{n}(\mathbb{F})$ denotes the $n \times n$ identity matrix. Then

$$
X \cdot Y=Y \cdot X
$$

and since the statement is assumed to be true for $n$,

$$
\phi(X)=\phi\left(\begin{array}{l|l}
A & \mathbf{0} \\
\hline \mathbf{0} & I
\end{array}\right)=l_{1}(\operatorname{det}(A)),
$$

where $\left.l_{1}:\right] 0,+\infty[\rightarrow \mathbb{R}$ is a logarithmic function.

Thus, if $X \in \mathscr{P}_{2 n}(\mathbb{R})$ has the form

$$
X=\left(\begin{array}{l|l}
A & \mathbf{0} \\
\hline \mathbf{0} & D
\end{array}\right),
$$

where $A, D \in \mathscr{P}_{n}(\mathbb{R})$, then

$$
\phi(X)=\phi\left(\begin{array}{c|c}
A & \mathbf{0} \\
\hline \mathbf{0} & D
\end{array}\right)=l_{1}(\operatorname{det}(A))+l_{2}(\operatorname{det}(D)),
$$

where $\left.l_{1}, l_{2}:\right] 0,+\infty[\rightarrow \mathbb{R}$ are logarithmic functions.

Let now $X \in \mathbb{P}_{n}(\mathbb{F})$ be arbitrary, further let $A$ and $D$ stand for the upper and lower $n \times n$ minors of $X$, that is,

$$
X=\left(\begin{array}{c|c}
A & B \\
\hline B^{*} & D
\end{array}\right)
$$

In this case $A, D \in \mathscr{P}_{n}(\mathbb{F})$.

Furthermore,

$$
\begin{aligned}
& \left(\begin{array}{c|c}
A & B \\
\hline B^{*} & D
\end{array}\right)=\left(\begin{array}{c|c}
\sqrt{A^{-1}} & 0 \\
\hline 0 & I
\end{array}\right) \cdot\left(\begin{array}{c|c}
I & 0 \\
\hline 0 & \sqrt{D^{-1}}
\end{array}\right) . \\
& \cdot\left(\begin{array}{c|c}
I & \sqrt{D^{-1}} \sqrt{A^{-1}} \sqrt{B} \sqrt{A^{-1}} \sqrt{D^{-1}} \\
\hline \sqrt{D^{-1}} \sqrt{A^{-1}} \sqrt{B} \sqrt{A^{-1}} \sqrt{D^{-1}} *
\end{array}\right) . \\
& \cdot\left(\begin{array}{c|c}
I & 0 \\
\hline 0 & \sqrt{D^{-1}}
\end{array}\right) \cdot\left(\begin{array}{c|c}
\sqrt{A^{-1}} & 0 \\
\hline 0 & I
\end{array}\right)
\end{aligned}
$$

Therefore, if we determine the function $\phi$ on elements having the form

$$
\left(\begin{array}{c|c}
I & B \\
\hline B^{*} & I
\end{array}\right) \quad\left(B \in \mathscr{M}_{n}(\mathbb{F})\right)
$$

then $\phi$ is completely determined. This can be done in a similar way as in the $2 \times 2$ case.

Part II. We will show that is the statement is true for $n \in \mathbb{N}$, then it is also true for $n-1$.

Assume that the statement is valid for a certain $n \in \mathbb{N}$, then $\mathscr{P}_{n-1}(\mathbb{R})$ can be embedded to $\mathscr{P}_{n}(\mathbb{R})$ through

$$
\mathscr{P}_{n-1}(\mathbb{R}) \ni A \longmapsto\left(\begin{array}{l|l}
A & \mathbf{0} \\
\hline \mathbf{0} & I
\end{array}\right) \in \mathscr{P}_{n}(\mathbb{R}) .
$$


Additionally, this embedding preserves the Jordan triple product. Define the $\operatorname{mapping} \widetilde{\phi}: \mathscr{S}_{n} \subset \mathscr{P}_{n}(\mathbb{R}) \rightarrow \mathbb{R}$ by

$$
\widetilde{\phi}\left(\begin{array}{c|c}
A & \mathbf{0} \\
\hline \mathbf{0} & 1
\end{array}\right)=\phi(A) \quad\left(A \in \mathscr{P}_{n-1}(\mathbb{R})\right),
$$

where

$$
\mathscr{S}_{n}=\left\{\left(\begin{array}{c|c}
A & \mathbf{0} \\
\hline \mathbf{0} & 1
\end{array}\right) \mid A \in \mathscr{P}_{n-1}(\mathbb{R})\right\} .
$$

Therefore, if for a mapping $\phi: \mathscr{P}_{n-1}(\mathbb{R}) \rightarrow \mathbb{R}$

$$
\phi(Y X Y)=\phi(X)+2 \phi(Y) \quad\left(X, Y \in \mathscr{P}_{n-1}(\mathbb{R})\right),
$$

then

$$
\phi(A)=\widetilde{\phi}\left(\begin{array}{c|c}
A & \mathbf{0} \\
\hline \mathbf{0} & 1
\end{array}\right)=l\left(\operatorname{det}\left(\left(\begin{array}{c|c}
A & \mathbf{0} \\
\hline \mathbf{0} & 1
\end{array}\right)\right)\right)=l(1 \cdot \operatorname{det}(A))=l(\operatorname{det}(A)),
$$

yielding that the statement is valid for $n-1$, as well.

\section{References}

[1] János Aczél, Lectures on functional equations and their applications., New York and London: Academic Press. XIX, 510 p. , 1966.

[2] Rajendra Bhatia, Positive definite matrices, Princeton Series in Applied Mathematics, Princeton University Press, Princeton, NJ, 2007. MR 2284176 (2007k:15005)

[3] Konstancja Bobecka and Jacek Wesołowski, Multiplicative Cauchy functional equation in the cone of positive-definite symmetric matrices, Ann. Polon. Math. 82 (2003), no. 1, 1-7. MR 2041392 (2005a:39049)

[4] Mirko Dobovišek, Maps from $M_{n}(\mathbb{F})$ to $\mathbb{F}$ that are multiplicative with respect to the Jordan triple product, Publ. Math. Debrecen 73 (2008), no. 1-2, 89-100. MR 2429028 (2009c:15021)

[5] Desmond Fearnley-Sander, A characterization of the determinant, Amer. Math. Monthly 82 (1975), no. 8, 838-840. MR 0399126 (53 \#2977)

[6] Gyula Gáspár, Die Charakterisierung der Determinanten über einem unendlichen Integritätsbereich mittels Funktionalgleichungen, Publ. Math. Debrecen 10 (1963), 244-253. MR 0168584 (29 \#5844)

[7] Sin Hitotumatu, A characterization of the determinant, Comment. Math. Univ. St. Paul. 13 (1964/1965), 45-50. MR 0178002 (31 \#2260)

[8] Marek Kuczma, An introduction to the theory of functional equations and inequalities. Cauchy's equation and Jensen's inequality. Edited by Attila Gilányi. 2nd ed., Basel: Birkhäuser. xiv, 595 p. , 2009.

[9] Svetozar Kurepa, On the characterization of the determinant, Glasnik Mat.Fiz. Astronom. Ser. II Društvo Mat. Fiz. Hrvatske 19 (1964), 189-198. MR 0179187 (31 \#3436)

[10] B. R. McDonald, A characterization of the determinant, Linear and Multilinear Algebra 12 (1982/83), no. 1, 31-36. MR 672914 (83j:15009) 
[11] Lajos Molnár, A remark on the Kochen-Specker theorem and some characterizations of the determinant on sets of Hermitian matrices, Proc. Amer. Math. Soc. 134 (2006), no. 10, 2839-2848 (electronic). MR 2231606 (2007b:81018)

[12] Kiyosi Yamaguti, Jordan and Jordan triple isomorphisms of rings, J. Sci. Hiroshima Univ. Ser. A 20 (1956/1957), 107-110. MR 0096710 (20 \#3193)

Eszter Gselmann

MTA-DE 'Lendület'

Functional Analysis Research Group

Institute of Mathematics

University of Debrecen

P. O. Box: 12 .

Debrecen

$\mathrm{H}-4010$

Hungary

e-mail: gselmann@science.unideb.hu 